\title{
The Study on Re Effect Correction for Laminar Wing with High Lift
}

\author{
Jieke Yao, Wenliang Feng, Lingying Lv and Bin Chen \\ Chengdu Aircraft Industrial (group) CO.LTD, 610092, Chengdu, China
}

\begin{abstract}
In the past years, Reynolds number (Re) effect correction is carried out by varied Re experiment. The experiment can obtain double logarithm linear curve between the least drag and Re, and the least drag in flying Re is got by linear exploration. However, Re effect exerts a great influence on transition position for laminar wing with high lift. Therefore, the lift, drag and longitudinal moment can't be acquired by traditional Re effect correction. In this paper, the transition prediction simulations for laminar wing high lift are carried to obtain unconstrained transition position in different $\mathrm{Re}$ by adopting $\gamma-\mathrm{Re}_{\theta}$ transition prediction model. An agreement with experiment for transition position is achieved. According to the result of transition perdition and unconstrained transition test, Re effect correction in different height for the data of wind tunnel test is done. This method makes up for the traditional method, which Re effect correction for battle-plane is done for only drag. In present paper, Re effect correction for the lift, drag and longitudinal moment is done to acquire more accurate experimental data to ensure everything in flight.
\end{abstract}

\section{Introduction}

Usually, the scaled-down model is adopted in the wind tunnel tests, which makes a greater difference between the wind tunnel tests and actual fight situation for the Reynolds number $(\mathrm{Re})$. Therefore, correction based on the Re effect for wind tunnel test data must be done.

Study on the Re effect of battle-plane shows that flow is almost all turbulence in the near boundary layer of the wings when it flies in the atmosphere. This attributes to apply thinner wings for the battle-plane. For such wings, Re has a smaller effect on the lift and longitudinal moment characteristics, but it exerts a powerful influence on the frictional drag on the surface of wing. Consequently, the Re effect correction is carried out for only the drag including the least drag $\left(C_{D \min }\right)$ and drag due to lift in the correction of wind tunnel data.

In order to pursue the higher cruising efficiency, civil aircraft and Unmanned Aerial Vehicle (UAV) having high height and speed properties in the modern world, such as supercritical aerofoil and supercritical laminar compound aerofoil, usually use high lift-drag ratio aerofoil. These aerofoils retain the natural laminar region at least $50 \%$ chord length in the designed cruising state. The research presents that the drag for laminar aerofoil is less than half usual turbulent aerofoil in the designed state. However, the natural laminar region disappears and the drag of aerofoil is rising rapidly when aerofoil deviates the designed state. And at the same time, the experimental research also shows that macroscopically aerodynamic characteristics containing the lift, drag and longitudinal moment for the supercritical aerofoil is influenced by Re because its flow structure and pressure distribution are changed by $\mathrm{Re}$ [1]. Transition position of supercritical aerofoil is also affected by Re. Transition which means transform from laminar flow to turbulence flow is very important concept in the boundary layer theory [2]-[5].

The correction method for Re effect of battle-plane is acquired by varied $\mathrm{Re}$ experiment. According to experiment, linearized curve, which $C_{D \min }$ varies according to $\mathrm{Re}$, is obtained. Therefore, $C_{D \min }$ for experiment $\mathrm{Re}$ is corrected to actual fight $\mathrm{Re}$ by extrapolation.

Numerical simulation based on $\gamma-\operatorname{Re}_{\theta}$ transition predicting mode for the wing is carried out in this paper, and the unconstrained transition position of wing in different $\mathrm{Re}$ is got. Meantime, forced transition test is carried out in the wind tunnel test, and aerodynamic data in the different transition position of wing is obtained. On the basis of results for the transition position of numerical calculation and data of unconstrained transition experiment, data correction based on Re effect for the lift and drag of wind tunnel test is done [6]-[11].

\section{The illustration of computation}

The commercial software ANSYS CFX, which is usually used by aerospace industry, is adopted for present study solving the Navier-Stokes (NS) equation. CFX is based finite-volume method of finite element, adopts coupled solution of full implicit-scheme and multi-mesh, and uses interpolation of 24 points for hexahedral mesh. Computational model employs hexahedral mesh in present paper, account of computational mesh is about 10 million. The geometry with grids is shown in the Fig. 1. 
No-slip boundary and opening boundary are respectively set up for solid boundary and far field boundary in this paper, and transition computation chooses Shear Stress Transport (SST) $\gamma-\mathrm{Re}_{\theta}$ model. The aim for transition model is solving intermittency function $\gamma(0 \leq \gamma \leq 1)$, which is chances of turbulence for the flow pattern of a point in space, and the aim for combination of transition model and SST turbulence model is controlling occurrence for the transition [12]-[14]. However, in some other similar works, even very recent studies, concerning on the Computational Fluid Dynamics (CFD) investigation of a fluid flow, they have employed other turbulence model such as the RNG-k- $\varepsilon$ [15], standard k- $\varepsilon$ model [16], bybrid URANS-LES [17].

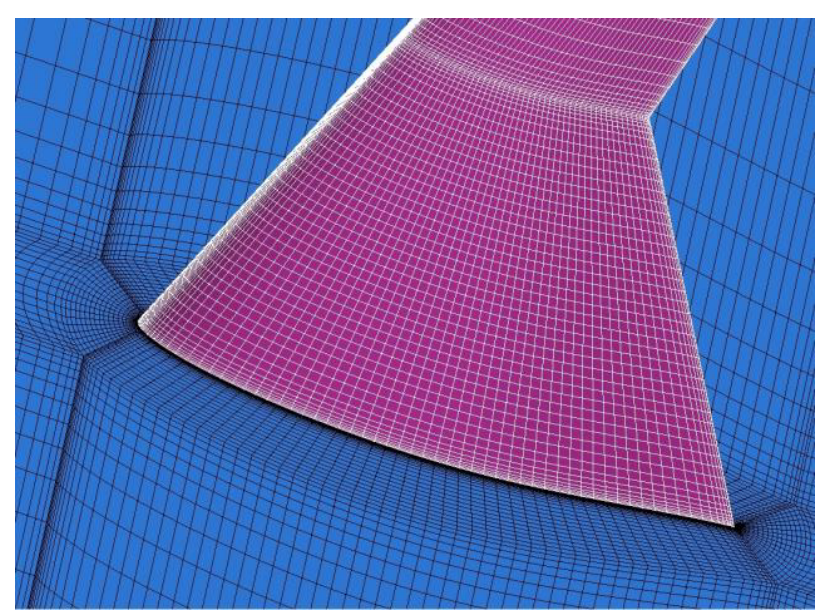

Figure 1. The geometry with grids

\section{Forced transition experiment}

In order to investigate the difference of aerodynamic characteristic on different transition position of wing, forced transition wind tunnel test is carried out by pasting special tape on the different position for the surface of wing. The results of experiments show that the changes, which $C_{D \min }$, angle of attack for 0 lift $\left(\alpha_{0}\right)$, slope of lift curve $\left(C_{L \alpha}\right)$ and longitudinal stability derivatives $\left(C_{m} C L\right)$ change with the transition position, basically presents linear. Re for the experiment is $1.23 \times 10^{6}, 1.51 \times 10^{6}$ and $1.76 \times 10^{6}$ for Mach number (Ma) of $0.4,0.5$ and 0.6 , respectively. The results are showed from Fig. 2 to Figure 6. From these figs, it is seen that aerodynamic data of different $\mathrm{Ma}$ presents certain difference in the same transition position, which mean that $\mathrm{Ma}$ exert an influence on the aerodynamic characteristic. Because different Ma reflects different experiment $\mathrm{Re}, \mathrm{Re}$ has an effect on aerodynamic data. And the influence of $R e$ is greater.

Forced transition experiment can't directly obtain data for the transition position of wing, and there are also more difficult to get transition position by unconstrained transition experiment. In this paper, some data, such as $\alpha_{0}, C_{L \alpha}, C_{D \min }$, are obtained by unconstrained transition experiment of full-aircraft, and the transition position of wing is acquired by linear extrapolation in the Fig. 2, Fig. 3 and Fig. 4.

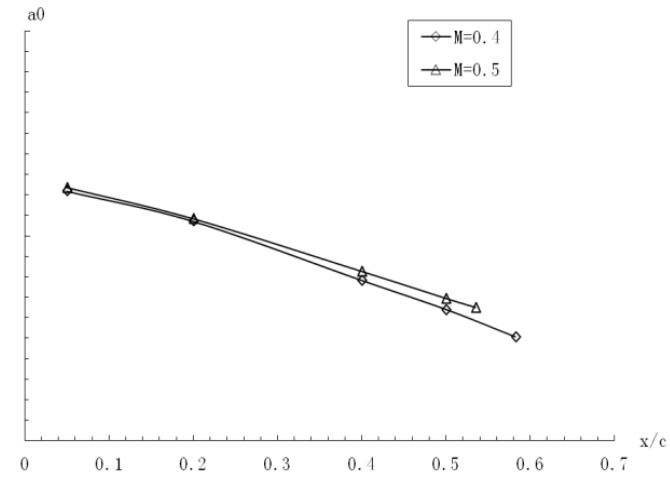

Figure 2. The relationship between $\alpha_{0}$ and transition position

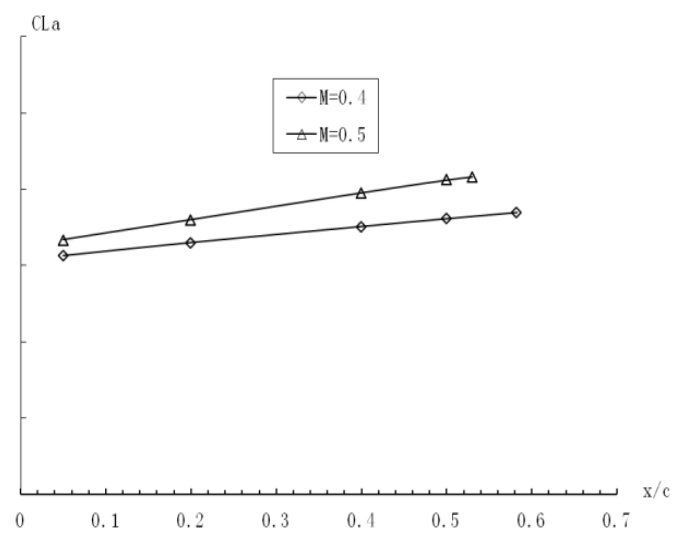

Figure 3. The relationship between $C_{L \alpha}$ and transition position

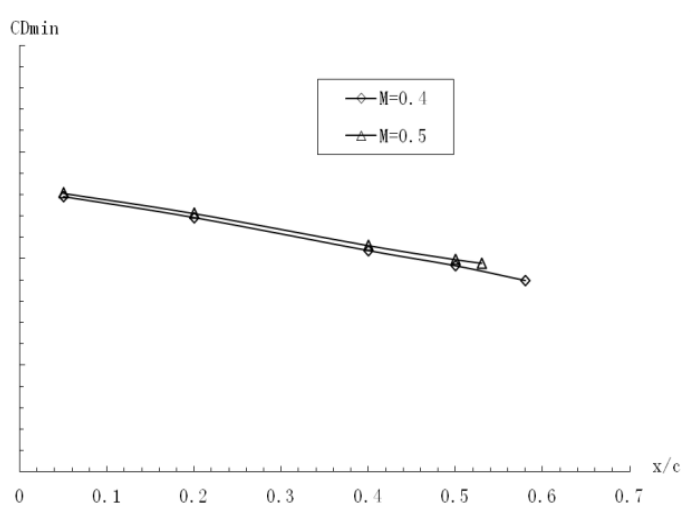

Figure 4. The relationship between $C_{D \min }$ and transition position

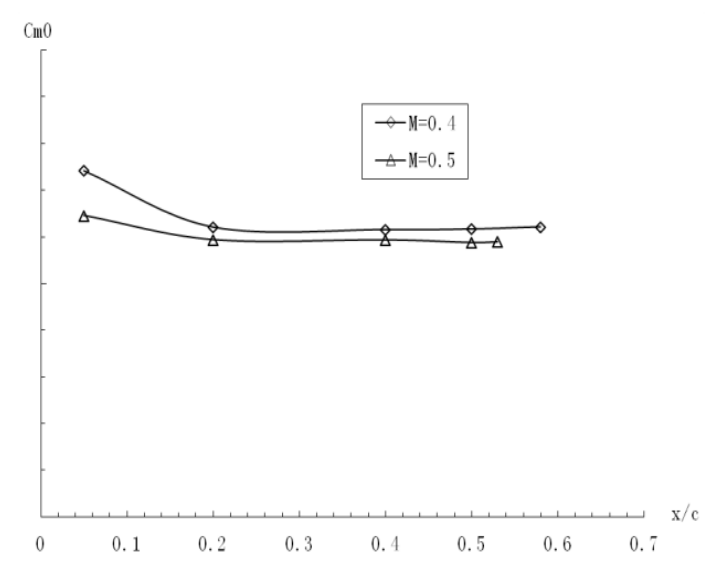

Figure 5. The relationship between moment for 0 lift $\left(C_{m 0}\right)$ and transition position 


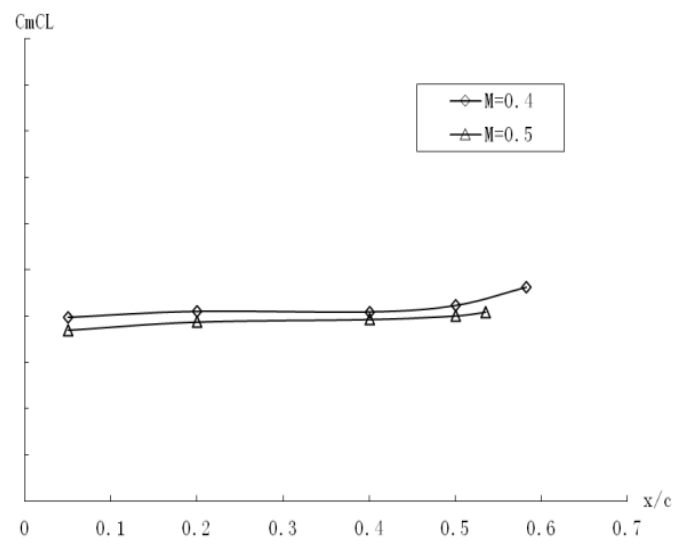

Figure 6. The relationship between $C_{m} C L$ and transition position

\section{Supercritical characteristic of wing}

In the initial design of wing, supercritical laminar compound airfoil was used to seek higher lift-drag ratio and critical Ma. In the later design, because the aircraft changed target and reduced flight speed, supercritical characteristic of wing wasn't used. Therefore, supercritical characteristic which is critical Ma wasn't fully verified in the wind tunnel test.

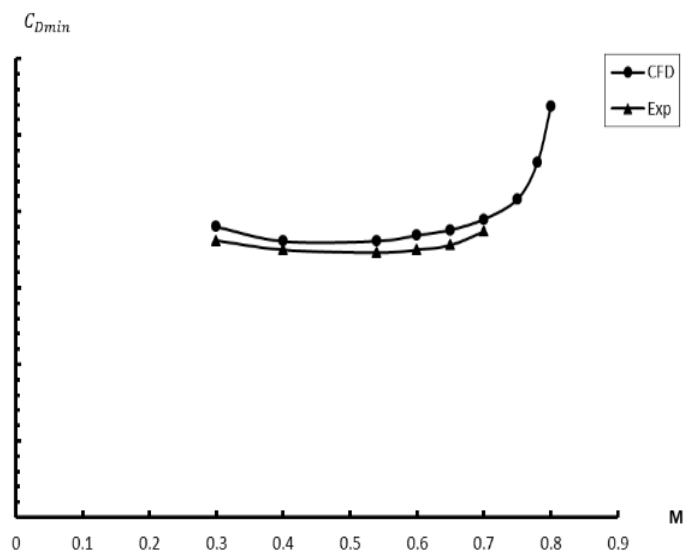

Figure 7. The comparison $C_{D \min }$ of wing between experiment and CFD

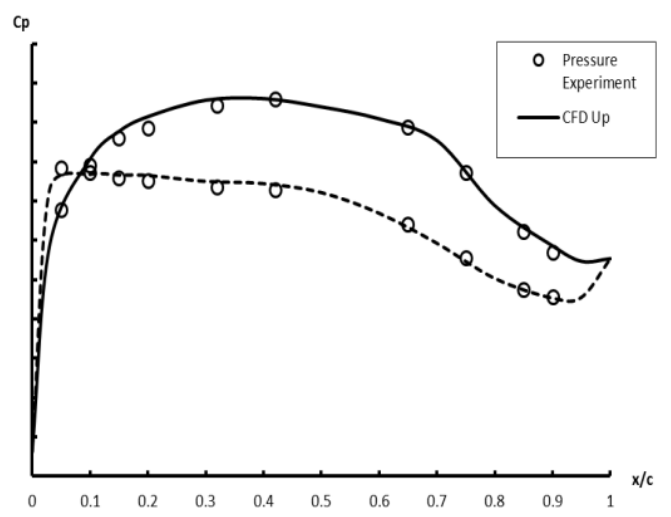

Figure 8. Pressure distribution of wing section $\left(\mathrm{M}=0.65, \alpha=0^{\circ}\right)$

The comparison of $C_{D \min }$ for the wind tunnel test and CFD simulations is shown in Figure 6. From the result of the Fig. 7, the critical Ma ( $\left.\partial C_{D \text { min }} / \partial M a=0.18\right)$ is about 0.8 . Fig. 8 and Fig. 9 show the comparison of pressure experiment and CFD simulation. That can be seen that the higher negative pressure doesn't present in the upper surface of the wing leading edge, pressure distribution is relatively flat in upper surface of middle wing. These can effectively weaken intensity of shock wave and increase the critical Ma. According to the above results, benign supercritical characteristic of wing can be presented.

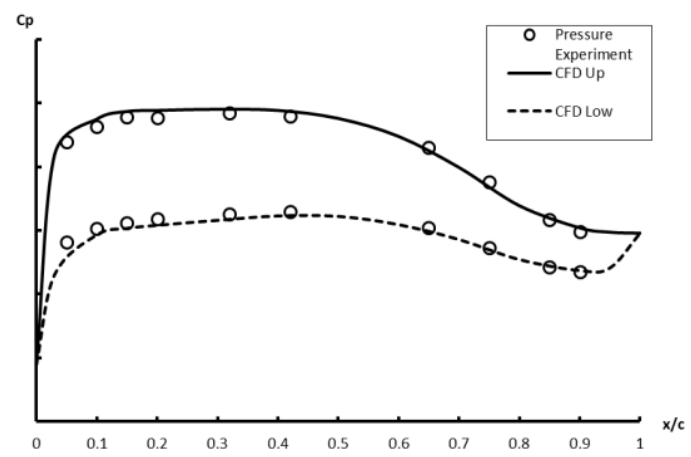

Figure 9. Pressure distribution of wing section $\left(M=0.65, \alpha=3^{\circ}\right)$

\section{The comparison of computation and experiment}

According to the result of forced transition for wing different position in the wind tunnel test, the experimental data for unconstrained transition position of wing is obtained by interpolation. Computational condition and experimental condition for unconstrained transition are identical.

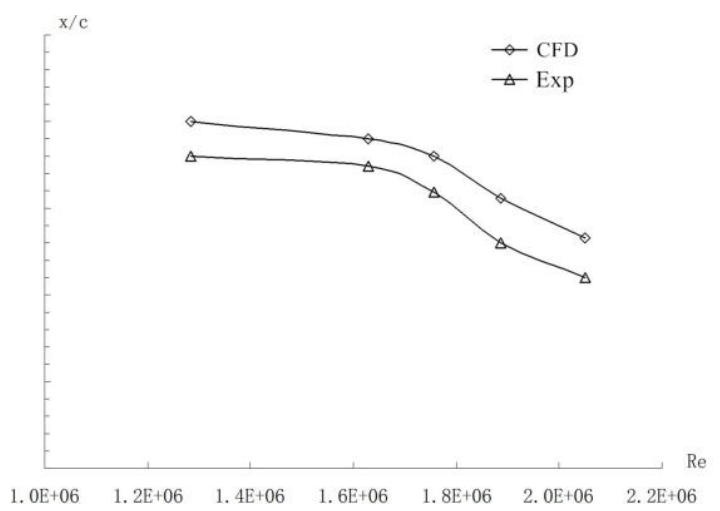

Figure 10. The comparison of transition position for CFD and experiment

Because Re change in a relatively smaller range in the wind tunnel test, the experimental Re is under $2.5 \times 106$. Fig. 10 gives the comparison of transition position for CFD simulation and the wind tunnel test. It can be seen that unconstrained transition position of wing for CFD simulation is back about $2 \%$ local chord than the tunnel test, varied trend, which transition position changes with $\mathrm{Re}$ is identical for CFD and experiment, and a great agreement is obtained.

Fig. 11, Fig. 12 and Fig. 13 show intermittent factor image on the upper surface of wing in different Re. It can be seen that intermittent factor is almost 0 , it immediately increases when reaching the transition position. 
Meantime, because of the influence of fuselage flow field, laminar range for inner section of wing is smaller than outer section of wing and the distribution of transition position along wingspan is no uniform.

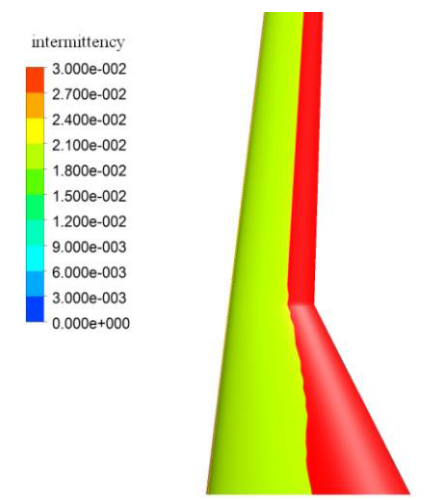

Figure 11. The distribution of intermittent factor $\left(\mathrm{Re}=2.0 \times 10^{6}\right)$

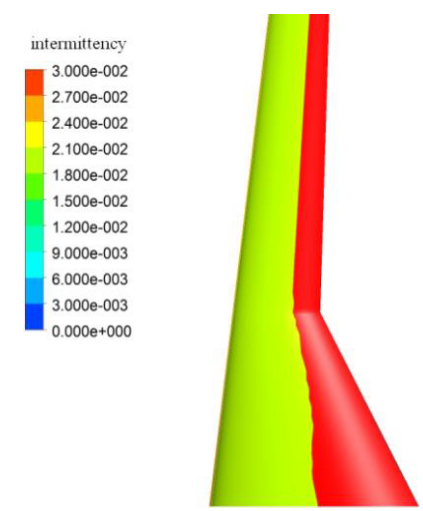

Figure 12. The distribution of intermittent factor $\left(\mathrm{Re}=5.0 \times 10^{6}\right)$

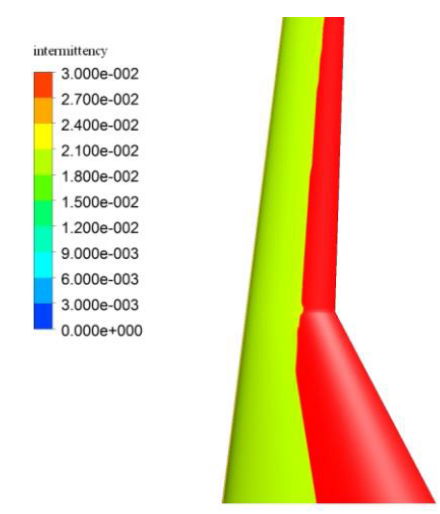

Figure 13. The distribution of intermittent factor $\left(\operatorname{Re}=8.0 \times 10^{6}\right)$

\section{The correction method for Re effect}

According to aircraft envelope, the range of flight $\mathrm{Re}$ is confirmed and numerical simulations for these Re are carried out to compute transition position in different height and speed. Because of deviation of $2 \%$ local chord for transition position of CFD and the wind tunnel test, the transition position for CFD is done certain correction.

Under the transition position of wing for different Re simulated by CFD and linear relation of transition position and $C_{D \min }, \alpha_{0}, C_{L \alpha}, C_{m 0}$ and $C_{m} C L$, aerodynamic derivatives in corresponding $\mathrm{Re}$ are obtained by linear interpolation (form Fig. 2 to Fig. 6). The formulas of correction are as follows.

$$
\begin{gathered}
C_{L}^{R e}=C_{L}^{e x p}+\Delta C_{L}^{R e} \\
\Delta C_{L}^{R e}=\Delta \alpha_{0}^{\text {forced transition }}+\Delta C_{L \alpha}^{\text {forced transition }} * \alpha \\
C_{D}^{R e}=C_{D}^{e x p}+\Delta C_{D}^{R e} \\
\Delta C_{D}^{R e}=\Delta C_{D m i n}^{\text {forced transition }} \\
C_{m}^{R e}=C_{m}^{e x p}+\Delta C_{m}^{R e} \\
\Delta C_{m}^{R e}=\Delta C_{m 0}^{\text {forced transition }}+\Delta C_{m C L}^{\text {forced transition }} * C_{L}^{R e}
\end{gathered}
$$

where $\quad \Delta \alpha_{0}^{\text {forced transition }} \quad, \quad \Delta C_{L \alpha}^{\text {forced transition }}$ $\Delta C_{D m i n}^{\text {forced transition }}, \Delta C_{m 0}^{\text {forced transition }}, \Delta C_{m C L}^{\text {forced transition }}$ are interpolated data in curve for forced transition experiment, respectively. According these experimental results, the derivatives for nonlinear range, such as $\Delta C_{L \alpha}^{\text {forced transitin }}$ and $\Delta C_{m C L}^{\text {forced transition, }}$ are corrected.

Unconstrained transition position computed in different $\mathrm{Ma}$ and $\mathrm{Re}$ is shown in Fig. 14 From this fig, it can be seen that while Re is smaller than $7 \times 10^{6}$, transition position is hardly changed, however, when Re is greater than $7 \times 10^{6}$, transition position is forward sharply and laminar range is shorten.

Experimental speed simulated for wind tunnel test is only similar to the real flight, but aerodynamic data in different height can't be obtained. Different height for flight of aircraft indicates different Re. Therefore, Re effect correction for the data for the wind tunnel test must be done to obtain aerodynamic data in different height of flight.

Fig. 15 and Fig. 16 show polar and pitching moment curve corrected for Re effect in different height. Fig. 17 gives the comparison of data for wind tunnel test corrected by $R e$ effect and aerodynamic data of flight test. The lift and drag of flight test are obtained by back-stepping for weight and thrust of engine for flight. From these results, an agreement is got for wind tunnel test data corrected by Re effect and flight test data, but certain differences are leaded to by thrust losing unable to obtain for flight data and data corrected.

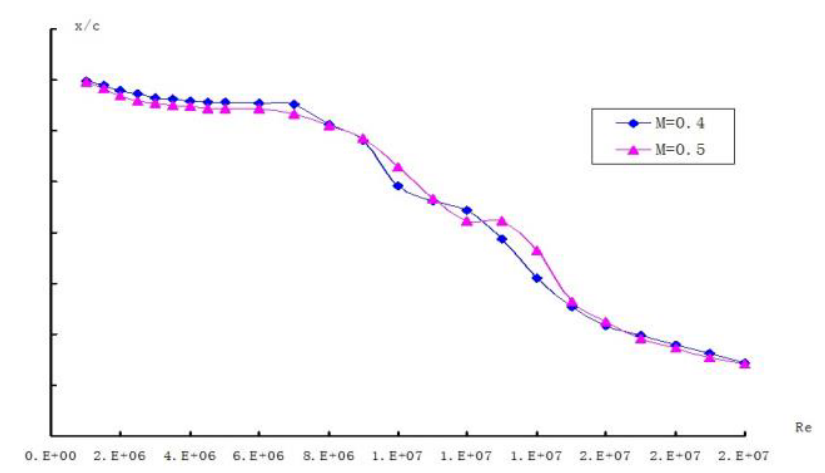

Figure 14. The relationship between transition position obtained by CFD and Re 


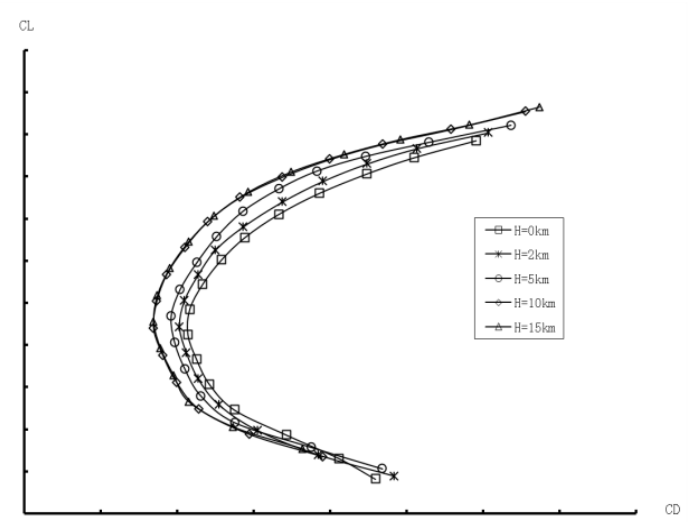

Figure 15. The polar of lift and drag corrected by Re effect

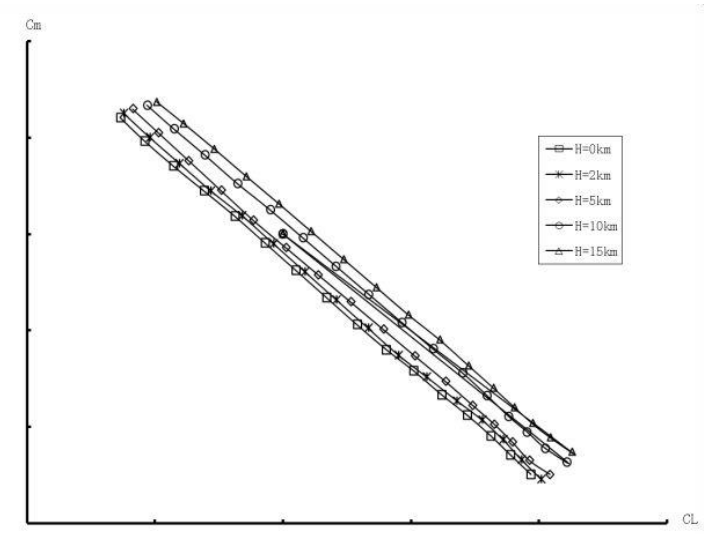

Figure 16. The curve of moment corrected by Re effect

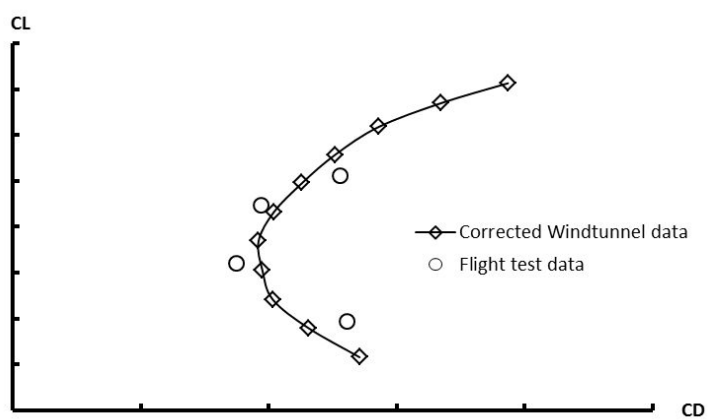

Figure 17. The comparison of polar of lift and drag for corrected by Re effect and flight test

\section{Conclusion}

According to the prediction for transition position and the method of $\mathrm{Re}$ effect correction for unconstrained transition experiment, more accurate and reliable data can be obtained. This corrected method has been applied in the aircraft of laminar wing with high lift and has been validated by experiment of flight. Present method may be used as reference to Re effect correction for data of wind tunnel test of the similar aircraft.

\section{References}

1. U. Frisch, B. Hasslacher, Y. Pomeau. "Lattice-gas automata for the Navier-Stokes equation". Physical Review Letters, 1986, vol. 56, pp. 1505-1508.
2. X. XU, D. W. Wei, D. H. Chen, Y. J. Wang. Analysis of flow over super-critical airfoil Reynolds number effects. CSTAM, 2012-B03-0140.

3. Y. C. Chen, J. T. Si, X. L. Han, J. L. Wei. Investigation of transition effect on the pressure distribution of super-critical wing. Acta Aerodynamica Sinica, 21 (4), pp. 470-475, (2003).

4. Q. Z. Yang, Z. Y. Zhang. Analysis of the boundary layer and aerodynamic characteristics of a supercritical laminar wing. Acta Aerodynamica Sinica, 25(5), pp. 439-422, (2004).

5. Y. Chen, Z. H. Gao. Application of gamma-theta transition model to flows around airfoils. Acta Aerodynamica Sinica, 27(4): 411-418, 2009.

6. D. B. DeGraff,D. R. Webster,J. K. Eaton. The effect of Reynolds number on boundary layer turbulence. Experimental Thermal and Fluid Science, 18: 341346, (1999).

7. S. Y,H. P. Modeling of flow transition using an intermittency transport equation. Journal of Fluids and Engineering, 122(2): 273-284, (2000).

8. Y. B. Suzen,G. Xiong, P. G. Huang. Prediction of transition flows in a low-pressure turbine using an intermittency transport equation. AIAA-2000-2645.

9. Y. L. Zhang, G. X. Wang, D. H. Meng, Y. T. Wang. Calibration of $\gamma-\operatorname{Re} \theta$ transition model. Acta Aerodynamica Sinica, 29(3): 295-301, (2011).

10. R. B. Langtry,F. R. Menter. Transition modeling for general CFD applications in aeronautics. AIAA paper, 2005-522.

11. R. E. The role of laminar-turbulent transition in gas turbine engines. ASME Journal of Turbo machinery, 113: 509-537, (1991).

12. R. B. Langtry, F. R. Menter, S. R. Likki, Y. B. Suzen, P. G. Iiuang, S. Volker. A correlation based transition model using local variables: part II-test case and industrial application. ASME-GT, 200453454.

13. F. R. Menter, R. B. Langtry, S. R. Likki, Y. B. Suzen, P. G. Iiuang, S. Volker. A correlation based transition model using local variables: part I-model formulation[R]. ASME-GT, 2004-53452.

14. R. B. Langtry. A correction-based transition model using local variables for unstructured parallelized CFD codes. Ph D Thesis, Stuttgart, (2006).

15. M. Miltner, M. Jordan. CFD simulation of straight and slightly swirling turblence free jets using difference RANS-turblence model[J]. Applied thermal engineering, $89: 1117-1126$, (2015).

16. B. Takabi, H. Shokouhmand. Effect of Al203/water hybrid nanofluid on heat transfer and flow characteristics in turbulent regime[J]. International journal of modern pyhsics, 26(4) : 1550047-1550050, (2015).

17. G. Vijayakumar, J. G. Brasseur, A. Lavely, B. Jayaraman, B. C. Craven. Interaction of atmospheric turbulence with blade boundary layer dynamics on a $5 \mathrm{MW}$ wind turbine using blade-boundary-layerresolved CFD with hybird URANS-LES[C]. 34th wind energy symposium, California, USA, (2016). 